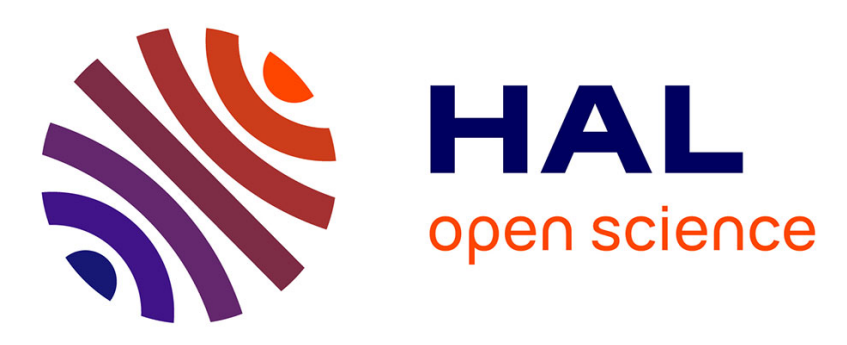

\title{
Effects of shock wave therapy in the skin of patients with progressive systemic sclerosis: a pilot study
}

Elisa Tinazzi, Ernesto Amelio, Elettra Marangoni, Claudio Guerra, Antonio

Puccetti, Orazio Michele Codella, Sara Simeoni, Elisabetta Cavalieri, Martina

Montagnana, Roberto Adani, et al.

\section{To cite this version:}

Elisa Tinazzi, Ernesto Amelio, Elettra Marangoni, Claudio Guerra, Antonio Puccetti, et al.. Effects of shock wave therapy in the skin of patients with progressive systemic sclerosis: a pilot study. Rheumatology International, 2010, 31 (5), pp.651-656. 10.1007/s00296-009-1339-z . hal-00594497

\section{HAL Id: hal-00594497 \\ https://hal.science/hal-00594497}

Submitted on 20 May 2011

HAL is a multi-disciplinary open access archive for the deposit and dissemination of scientific research documents, whether they are published or not. The documents may come from teaching and research institutions in France or abroad, or from public or private research centers.
L'archive ouverte pluridisciplinaire HAL, est destinée au dépôt et à la diffusion de documents scientifiques de niveau recherche, publiés ou non, émanant des établissements d'enseignement et de recherche français ou étrangers, des laboratoires publics ou privés. 
Effects of Shock Wave Therapy in the skin of patients with Progressive Systemic Sclerosis: a pilot study.

Elisa Tinazzi $\mathrm{MD}^{1^{*}}$, Ernesto Amelio $\mathrm{MD}^{2^{*}}$, Elettra Marangoni $\mathrm{MD}^{1}$, Claudio Guerra $\mathrm{MD}^{2}$, Antonio Puccetti MD ${ }^{3}$, Orazio Michele Codella $\mathrm{MD}^{1}$, Sara Simeoni MD ${ }^{1}$, Elisabetta Cavalieri $\mathrm{PhD}^{4}$, Martina Montagnana $\mathrm{MD}^{5}$, Roberto Adani $\mathrm{MD}^{2}$, Roberto Corrocher $\mathrm{MD}^{1}$ and Claudio Lunardi $\mathrm{MD}^{1}$.

${ }^{1}$ Department of Clinical and Experimental Medicine, University of Verona, P.le L.A. Scuro 10, 37134 Verona, Italy

2 Shock Wave Unit-Hand Surgery Section, University Hospital of Verona, P.le L.A.Scuro 10, 37134 Verona, Italy

3 Department of Experimental medicine, University of Genova and Institute G. Gaslini, L.go G. Gaslini 5, 16148 Genova, Italy

${ }^{4}$ Department of Morphological and Technical Sciences, University of Verona, St. Le Grazie 8, 37134 Verona

${ }^{5}$ Department of Morphological and Biomedical Sciences, University of Verona, P.le L.A.Scuro 10, 37134 Verona, Italy

* These two authors equally contributed to the work

Corresponding Author:

Professor Claudio Lunardi

Department of Clinical and Experimental Medicine, University of Verona, P.le L.A. Scuro 10, 37134 Verona, Italy

Phone number: +390458124759

Fax n.: +39-045-8027473

E-mail: claudio.lunardi@univr.it 


\begin{abstract}
Objectives: Vasculopathy, immunological abnormalities and excessive tissue fibrosis are key elements in the pathogenesis of progressive systemic sclerosis (SSc). Extracorporeal shock waves (ESW) have anti-inflammatory and regenerative effects on different tissues. We hypothesized that ESW can reduce endothelial cell damage and skin fibrosis in patients with SSc.
\end{abstract}

Methods: We enrolled 30 patients affected by SSc, 29 females and 1 male. Rodnan Skin Score (RSS) and Visuo-Analogical Scale (VAS) for skin wellness were performed before and immediately after ESW therapy (ESWT) and at 7, 30, 60 and 90 days after the treatment. Sonographic examination of the patients' arms was performed before and 7, 30, 60, 90 days after treatment. Blood samples were obtained before and 30 and 60 days after treatment to measure serological levels of von Willebrand factor, vascular endothelial growth factor, intracellular adhesion molecule-1, monocyte chemotactic protein-1; the number of endothelial progenitor cells (EPCs) and circulating endothelial cells (CECs) were determined at the same time points.

Results: After ESWT we observed a rapid and persistent reduction of RSS and decrease of VAS. There was no difference in skin thickness before and after ESWT; however we observed a more regular skin structure and an improvement in skin vascularization 90 days after treatment. EPCs and CECs increased 60 and 90 days after treatment, while serological biomarkers showed no variation before and after therapy.

Conclusions: ESWT resulted in an improvement of VAS, RSS and of skin vascular score. An increase of CECs and EPCs was also observed after therapy.

Key words: ESWT: extracorporeal shock wave therapy; SSc: progressive systemic sclerosis; skin fibrosis; endothelial cell damage. 


\section{Introduction}

Progressive systemic sclerosis ( $\mathrm{SSc}$ ) is an autoimmune disease characterized by excessive deposition of collagen in the skin and visceral organs. Pathogenesis of the disease is complex: the pivotal steps are endothelial cell damage, immune activation and collagen production by fibroblasts [1]. Endothelial involvement is associated with the increase of some circulating markers including von Willebrand factor (vWF), vascular endothelial growth factor (VEGF), intracellular adhesion molecule-1 (ICAM-1), monocyte chemotactic protein-1 (MCP-1) [2,3].

The treatment of SSc is still disappointing since it is not able to modify the course of the disease. Conventional therapies are directed to improve peripheral blood circulation, to prevent the synthesis and release of harmful cytochines and possibly to inhibit or reduce fibrosis [4].

Extracorporeal shock waves (ESW) are defined as a sequence of sonic pulses characterized by high peak pressure (up to $100 \mathrm{MPa}$ ), fast pressure rise (10-100 ns) and short lifecycle. First applied in 1980 for the treatment of kidney stones, during the last 10 years this tecnique was found to induce an immediate anthalgic and antiinflammatory effect and a long-term tissue regeneration together with increase of angiogenesis [5-9].

ESW have found widespread use in orthopaedics. ESW have a positive influence on both calcifying tendonitis of the shoulder and fracture healing [10-11]. Moreover, low-energy shock waves therapy is used for persistent tennis elbow syndrome and painful heel with significant positive clinical results [12-13].

Recently Nishida et al performed extracorporeal shock wave application on the ischemic myocardial region ( 200 shots/spot for 9 spots at $0,09 \mathrm{~mJ} / \mathrm{mm}^{2}$ ) in a porcine model of chronic myocardial ischemia [14]. The cardiac shock wave therapy intervention improved global and regional myocardial functions in the treated animals as well as regional perfusion measured as myocardial blood flow of the chronic ischemic region without any adverse effects. No rise in $\mathrm{CK}, \mathrm{CkmB}$ or Troponin was observed in that study. Vascular density increased in the shock wave 
treated area and VEGF production was enhanced in the ischemic myocardium in vivo [14].

The aim of this work was to investigate whether ESWT applied to limited skin area might reduce fibrosis and increase vascularization in patients affected by systemic sclerosis.

\section{Matherials and methods}

\section{Patients selection}

From January to April 2008 we enrolled 30 patients affected by systemic sclerosis, 29 female and 1 male, aged 27-76 years old (mean age 55.9 yrs), 19 with limited cutaneous disease, 10 with diffuse cutaneous disease and 1 with overlap syndrome polymiositis/systemic sclerosis; disease duration varied from 11 months to 23 years (mean duration 6,7 yrs).

All patients were treated with calcium channel blockers and antiplatelet aggregation drugs (acetyl-salicylic acid) and monthly infusion of prostanoids for 8 hours.

Written informed consent was obtained from all patients before entering the study.

\section{Treatment regimen}

An electromagnetic lithotriptor (DUOLITH SD1 device; Storz Medical AG, Switzerland) was used for ESWT. The electromagnetic generator of the device consists of a cylindrical wire wound coil, a metallic membrane and a concentric paraboloid reflector. Swithching an electrical pulse in the kilo amperes range to a cylindrical coil which is surrounded by the metallic membrane, strong eddy currents are induced and the membrane is elongated, emitting a cylindrical acoustic wave in water. This wave is not yet a shock wave. It is focused by the reflector and it is steepening on its way to the focus.

Treatment protocol consisted of three sittings. The pressure pulses were focused on the volar and dorsal side of the forearm and on the hand and the fingers of one upper 
arm, following this protocol: 2000 shots along the dorsal forearm, 2000 shots along the volar forearm, 1000 shots along the dorsal side of the hand and fingers, 1000 shots along the volar side of the hand and fingers. The other upper arm and hand were used as controls. Defocused energy applied was $0.20 / 0.25 \mathrm{~mJ} / \mathrm{mm}^{2}$ with a repetition frequency of 4Hz. Treatment did not require any kind of anaesthetic.

Twenty-eight patients completed the entire treatment, while two patients received only two sittings.

\section{Clinical evaluation}

Skin involvement was determined by the modified Rodnan skin score (mRSS) [with palpation of 17 anatomical sites and scoring on a 0-3 scale, where $0=$ normal skin, $1=$ slight thickening, $2=$ moderate thickening, $3=$ hidebound skin sclerosis $]$.

A visual-analogic scale (VAS) was used to asses skin wellness; it assessed skin elasticity and softness and oedema, sensitivity and pain of the hand scoring on a 0 100 scale $(0=$ the best possible condition, $100=$ the worst possible condition)

RSS and VAS were performed before, immediately after the first ESWT and then at $7,30,60,90$ days after the end of the treatment.

\section{Ultrasonographic evaluation}

Skin thickness and vascularity were measured with a high frequency ultrasound scanner (LOGIQ Book XP ultrasound machine; GE Healthcare, UK) using a $12 \mathrm{MHz}$ transducer (I12L) and an ultrasound pad to increase ultrasonographic signal. Scans were obtained form volar and dorsal side of upper harm $10 \mathrm{~cm}$ distal from elbow; total skin thickness (expressed in centimetres) was calculated as mean of these two measures. Skin vascularity was obtained by colorDoppler analysis and represented on an arbitrary scale ranging from 1 (scarse) to 3 (elevate); total vascular score was the mean of values obtained for dorsal and volar side of upper harm.

Sonographic examination was performed before ESWT and at 7, 30,60 and 90 days after the end of the treatment. 
Biochemicals markers and circulating endothelial cells

Blood samples were obtained before and 30,60 days after the end of ESWT. Among the markers of endothelial cell disfunction we have tested vWF, VEGF, ICAM-1 and MCP-1 which is also a marker of fibrosis. vWF-Ag was measured by an automated quantitative enzyme linked immunosorbent assay (ELISA) on the mini Vidas (BioMeriuex, Marcy L'Etoile, France). Determination of VEGF (R\&D System Quantikine (®) Minneapolis, MN, USA), MCP-1 and ICAM-1 (Endogen Thermo $\underline{\text { Scientific }(\text {; } \text { Rockford, Illinois, USA) were carried out using commercially available }}$ kits following the manufacturer's instructions.

The number of endothelial progenitor cells (EPCs) and circulating endothelial cells (CECs) was determined at the same time points. CECs and EPCs were detected by flow-cytometry by lyse-no-wash method. Two hundred $\mu \mathrm{L}$ of each sample were incubated with a panel of monoclonal antibodies for 20 minutes at room temperature. Fluorescein isothiocyanate (FITC)-conjugated anti-CD45 (10 $\mu$ l), R-Phycoerythrin (PE)-conjugated anti-CD34,-CD31 and -CD146 (10 $\mu \mathrm{l})$ or isotype-matched control $\left(\operatorname{IgG}_{1}\right)$, allophyco-cyanine (APC) anti-CD3,-CD16, -CD19 and -CD33 (5 $\left.\mu 1\right)$ were used. 7-amino-actinomycin (7-AAD) was added for dead cells exclusion. Samples were also stained with anti-CD45 FITC, anti-CD34, -CD31 and -CD146 PE, antiCD106 or anti-VEGFR2 APC and peridin chlorophill protein (PerCP)-conjugated anti-CD3,-CD16, -CD19 and -CD33. All reagents were purchased from Becton Dickinson (San Jose, CA, USA), except for anti-CD16 (Caltag, Burlingame, CA, USA), anti-CD106 (Biolegend, San Diego, CA, USA) and anti-VEGFR2-APC (R\&D System, Minneapolis, MN, USA). After labeling, red blood cells were lysed by incubation with $2 \mathrm{ml}$ of Ammonium Chloride lysis solution and then the sample was analysed on a FACS Calibur cytometer (Becton Dickinson). The sensitivity of fluorence detectors was set and monitored using Calibrite Beads (Becton Dickinson) according to the manufacturer's recomendations; 500.000 cells per sample were acquired in live gating. Data were analyzed with CellQuest software (Becton 
Dickinson). Appropriate analysis gates, designed to remove dead cells, platelet aggregates and debris, and to exclude CD45+ and CD3+/CD16+/CD19+/CD33+ hematopoietic cells (dump channel), were used to enumerate total CECs and EPCs .

\section{Nitric Oxide evaluation}

In 3/30 patients levels of nitric oxide were measured before and during ESWT at time 2 and 4 minutes after beginning of treatment. Enzymatic Griess assay on deproteinated serum was used to determine nitric oxide values (kit Cayman Chemical; Ann Arbor, Michigan, USA) [15].

\section{Statystical analysis}

Calculations were performed with the SPSS16 statistical package. For statistical analysis Student's $t$ test was used and a $p<0,05$ was considered statistically significant.

\section{$\underline{\text { Results }}$}

\section{Clinical evaluation}

$\underline{\text { Rodnan Skin Score showed a statistically significant reduction }(p<0,001)}$ immediately after the first sitting of ESWT and 7 and 30 days after treatment compared to the basal value, while VAS showed a statistically significant decrease at all time points ( $\mathrm{p}=0,0390$ days after the end of treatment), as shown in table 1.

\section{Ultrasonographic evaluation}

No significant changes were observed in both skin thickness and vascularity at time 7, 30, 60 and 90 days after ESW therapy compared to the basal scores. However we observed a more regular skin structure, as shown in figure 1. Moreover, the vascular score 90 days after treatment was increased compared to the basal score nearly 
reaching a statistical significant difference $(\mathrm{p}=0.06)$. Table 2 shows the results of ultrasonographic examination.

\section{Biochemical markers and circulating endothelial cells}

The baseline evaluation of biochemical parameters gave the following results: vWF $103.3 \pm 54.9 \mathrm{mg} / \mathrm{L}$, VEGF $710.4 \pm 592.1 \mathrm{pg} / \mathrm{mL}$, ICAM-1 $291 \pm 139.1 \mathrm{ng} / \mathrm{mL}$, MCP-1 $323.7 \pm 213 \mathrm{pg} / \mathrm{mL}$. No significant changes were observed at time 30 and 60 days after the end of treatment (table 3 ).

Both EPCs and CECs showed a significant increase 30 and 60 days after the end of treatment compared to the basal $(\mathrm{p}<0,05)$ as shown in table 4 .

\section{Nitric Oxide evaluation}

In 2 out of the 3 patients examined nitric oxide dosage showed an increase after 2 minutes from the beginning of ESW therapy (data not shown).

\section{Discussion}

The present study demonstrates that, in patients affected by SSc, skin application of extracorporeal shock waves causes a rapid and persistent improvement of clinical parameters (RSS and VAS for skin wellness) and a late increase in skin vascularization and in number of EPCs and CECs.

Shock waves were used since 1980's to treat kidney stones. During the last 10 years this tecnique was found to induce an immediate anthalgic and anti-inflammatory effect and a long-term tissue regeneration together with increase of angiogenesis [5-9, 16]. One of the possible mechanisms of action of the anti-inflammatory effect of ESWT may be related to the ability of ESW to keep local NO contents at a physiological level in the early phase of inflammatory response, enhancing either a non-enzymatic or enzymatic production of NO [15]. Therefore induction of NO 
synthesis has been suggested to be one of the most important mechanism implicated in the anti-inflammatory effect of ESWT, while increased expression of VEGF and the consequent mobilization of endothelial progenitor cells can explain the proangiogenic action $[6-8,17]$. It has been recently demonstrated that, in addition to angiogenesis due to migration and proliferation of endothelial cells in situ, EPCs contribute to neovascularization in ischemic tissue through a vasculogenetic mechanism and through secretion of a variety of angiogenic factors [18-19]. Finally, recent studies have marked out that ESW are able to recruit stem cells and to stimulate their differentiation in various damaged tissues inducing reparative phenomena [20-22].

On these scientific bases, we studied the effect of low-energy ESWT on skin and serological biomarkers in patients affected by SSc.

Our results showed both a short-term and a long-term effect of ESW: we observed a rapid improvement of skin elasticity as measured by RSS and VAS, with a persistent effect during the time (until 30 days after the treatment for RSS and until 90 days for VAS).

Ultrasonographic evaluation of the skin showed no significant difference of skin thickness before and after ESWT, and after ESWT compared to the untreated controlateral arm. However we observed a regularization of skin structure with more defined skin layers; the lack of statistical significance is probably due to the limited number of patients analyzed. Moreover the modification of the skin structure may be responsible for the improvement of RSS and VAS. Finally we are evaluating whether a modification in skin thickness is detectable by ultrasonography in a longer period of observation.

The increasing of vascular score 90 days after the end of treatment is concordant with the hypothesis of ESW-induced neoangiogenesis. However, our study did not demonstrate an increase in serological levels of VEGF or a decrease of other biomarkers (vWF, MCP-1, ICAM-1) indicative of endothelial cell damage; this is possibly related to the application of ESW to a very limited skin area. On the contrary 
the pro-angiogenetic and reparative effect of ESW is demonstrated by the persistent increase of CECs and EPCs, which remains elevated until 60 days from the end of treatment.

Nitric oxide measurement was limited to only three patients because of difficulties in maintaining a venous access during ESW sitting. The increased values found in 2/3 patients is in accordance with literature $[6,7,17]$, but further studies are needed.

For all paramethers considered, we found no significant difference both between patients with limited and diffuse skin involvement or between patients with or without digital ulcerations, although the absence of differences may depend on the limited number of patients enrolled in each subgroup.

In conclusion, the results of our study suggest that ESWT is a novel and efficacious treatment that can be added to the pharmacological therapy in order to decrease endothelial cell damage and skin fibrosis in patients affected by SSc. This treatment is well tolerated and can be repeated without side effects; in the majority of cases it determines a rapid improvement of skin elasticity and skin wellness, even if the effects tend to reduce during the time.

We are now planning to evaluate the time of retreatment and the effects of ESWT applied to more extensive skin areas, such as face and neck, to ameliorate both functional and aesthetic aspects. Moreover we are performing skin biopsy with immunohistochemical analysis prior to and after ESWT.

\section{Acknowledgment}

We thanks Dr. Ernst Marlinghaus for his invaluable technical support. 


\section{References}

1. Abraham DJ and Varga J (2005) Scleroderma: from cell and molecular mechanisms to disease models. TRENDS Immunol; 26: 587-595.

2. Matucci Cerinic M, Valentini G, Sorano GG et al (2003) Blood coagulation, fibrinolysis and markers of endothelial dysfunction in systemic sclerosis. Arthritis Rheum; 32: 285-295.

3. Carvalho JF, Blank M, Shoenfeld Y (2007) Vascular endothelial growth factor (VEGF) in autoimmune disease. J Clin Immunol 27:246-256.

4. Denton CP, Black CM (2005) Targeted therapy comes of age in scleroderma. TRENDS Immunol 26: 596-602.

5. Meier R, Kamelger FS, Piza-Katzer H (2005) Shock wave therapy: an innovative treatment method for partial thickness burns. Burns 31:921-922.

6. Mariotto S, Cavalieri E, Amelio E et al (2005) Extracorporeal shock waves: from lithotripsy to anti-inflammatory action by NO production. Nitric oxide 12: 89-96.

7. Ciampa AR, Carcereri de Prati A, Amelio E et al (2005) Nitric oxide mediates anti-inflammatory action of extracorporeal shock waves. FEBS Letters 579: 6839-6845.

8. Aicher A, Heeschen C, Sasaki K et al (2006) Low-energy shock wave for enhancing recruitment of endothelial progenitor cells. A new modality to increase efficacy of cell therapy in chronic hind limb ischemia. Circulation 114: 2823-2830.

9. Schaden W, Thiele R, Kölpl C et al (2007) Shock wave therapy for acute and chronic soft tissue wounds: a feasibility study. J Surg Res 143: 1-12.

10. Rompe JD, Rumler F, Hopf C, Nafe B, Heine J (1995) Extracorporeal shock wave therapy for calcifying tendonitis of the shoulder. Clin Orthop 321: 196201.

11. Haupt G, Haupt A, Ekkernkamp A, Gerety B, Chvapil M (1992) Influence of shock waves on fracture healing. Urology 39: 529-532. 
12. Rompe JD, Hopft C, Kullmer K, Heine J, Burger R, Nafe B (1996) Low energy extracorporeal shock wave therapy for persistent tennis elbow. Int Orthop 20: 23-27.

13. Rompe JD, Hopft C, Nafe B, Burger R (1996) Low-energy extracorporeal shock wave therapy for painful heel: a prospective controlled single-blind study. Arch Orthop 1996; 115: 75-79.

14. Nishida T, Shimokava H, Keiji O, et al (2004) Extracorporeal cardiac shock wave therapy markedly ameliorates ischemia-induced myocardial dysfunction in pigs in vivo. Circulation 110: 3055-3061.

15. Romitelli F, Santini SA, Chierici E et al (2007) Comparison of nitrite/nitrate concentration in human plasma and serum samples measured by enzymatic batch Griess assay, ion-pairing HPLC and ion-trap GC-MS: the importance of correct removal of proteins in Griess assay. J Crom B 851: 257-267.

16.Wang FS, Wang CJ, Chen YJ et al (2004) Ras induction of superoxide activates ERK-dependent angiogenic transcriptional factor HIF-1 and VEGF-A expression in shock wave-stimulated osteoblasts. J Biol Chemistry 279: 1033110337.

17. Mariotto S, Carcereri de Prati A, Cavalieri E et al (2009) Extracorporeal shock wave therapy in inflammatory diseases: molecular mechanism that triggers anti-inflammatory action. Curr Med Chem 16: 2366-2372.

18. Urbich C, Aicher A, Heeschen C et al (2005) Soluble factors released by endothelial progenitors cells promote migration of endothelial cells and cardiac resident progenitor cells. J Mol Cell Cardiol 39: 733-742.

19. Yan X, Zeng B, Chai Y, Luo C, Li X (2008) Improvement of blood flow, expression of nitric oxide, and vascular endothelial growth factor by lowenergy shock wave therapy in random-pattern skin flap model. Ann Plast Surg $61 ; 646-653$.

20. Chen YJ, Wurtz T, Wang CJ et al (2004) Recruitment of mesenchimal cells and expression of TGF- $\beta 1$ and VEGF in the early stage of shock wave- 
promoted bone regeneration of segmental defects in rats. J Orthop Res 22: 526534.

21. Wang SF, Yang KD, Chen RF et al (2002) Extracorporael shock-wave promotes growth and differentiation of bone marrow stromal cells towards osteoprogenitors associated with induction of TGF- $\beta 1$. J Bone Joint Surg $\mathrm{Br}$ 84: 457-461.

22. Nurzynska D, Di Meglio F, Castaldo C et al (2008) Shock waves activate in vitro cultured progenitors and precursors of cardiac cell lineages from the human heart. Ultrasound Med Biol 34: 334-342. 
Table 1. Rodnan Skin Score (RSS) and Visual-analogic Scale (VAS) before and after ESW therapy.

\begin{tabular}{|c|c|c|}
\hline Measure & RSS & VAS $(0-100)$ \\
\hline Pre-ESWT & $28.62 \pm 7.56$ & $47.2 \pm 14.9$ \\
\hline After Ist sitting & $27.14 \pm 8.24 *$ & $27.2 \pm 13.3 *$ \\
\hline After 7 days & $27.98 \pm 6.29 *$ & $31.4 \pm 16.8 *$ \\
\hline After 30 days & $28.09 \pm 6.30 *$ & $31.4 \pm 17.3 *$ \\
\hline After 60 days & $28.21 \pm 6.14$ & $40.7 \pm 18.1 *$ \\
\hline After 90 days & $27.96 \pm 7.25$ & $42.8 \pm 16.2 *$ \\
\hline
\end{tabular}

$* \mathrm{p}<0,05$

Table 2. Skin thickness and vascular score measured by ultrasound.

\begin{tabular}{|c|c|c|}
\hline Measure & Skin thickness $(\mathrm{cm})$ & Vascular score \\
\hline $\begin{array}{c}\text { Pre-ESWT and } \\
\text { controlateral untreated } \\
\text { arm (any time point) }\end{array}$ & $2.7 \pm 0.3$ & $1.78 \pm 0.65$ \\
\hline After 7 days & $2.6 \pm 0.3$ & $1.76 \pm 0.51$ \\
\hline After 30 days & $2.6 \pm 0.2$ & $1.62 \pm 0.58$ \\
\hline After 60 days & $2.5 \pm 0.3$ & $1.55 \pm 0.47$ \\
\hline After 90 days & $2.6 \pm 0.3$ & $1.42 \pm 0.37^{*}$ \\
\hline
\end{tabular}

$* \mathrm{p}=0,06$ 
Table 3. Biochemical markers measured before and after ESWT.

\begin{tabular}{|c|c|c|c|c|}
\hline Measure & $\begin{array}{c}\text { VWF } \\
(\mathrm{mg} / \mathrm{L})\end{array}$ & $\begin{array}{c}\text { sVEGF } \\
(\mathrm{pg} / \mathrm{mL})\end{array}$ & $\begin{array}{c}\text { sICAM-1 } \\
(\mathrm{ng} / \mathrm{mL})\end{array}$ & $\begin{array}{c}\text { sMCP-1 } \\
(\mathrm{pg} / \mathrm{mL})\end{array}$ \\
\hline Pre-ESWT & $103.3 \pm 54.9$ & $710.4 \pm 592.1$ & $291 \pm 139.1$ & $323.7 \pm 213$ \\
\hline After 30 days & $121.6 \pm 111$ & $698.6 \pm 382.2$ & $279.4 \pm 116.8$ & $408.1 \pm 185.4$ \\
& & & & \\
\hline After 60 days & $98.7 \pm 65.3$ & $702 \pm 370.5$ & $290.9 \pm 129.7$ & $396.7 \pm 196.4$ \\
& & & & \\
\hline
\end{tabular}

Table 4. Number of Endothelial Progenitors Cells (EPCs) and Circulating Endothelial Cells (CECs) before and after ESW therapy.

\begin{tabular}{|c|c|c|}
\hline Measure & CECs $/ \mathrm{mm}^{3}$ & $\mathrm{EPCs} / \mathrm{mm}^{3}$ \\
\hline Pre-ESWT & $586 \pm 356$ & $121 \pm 86$ \\
\hline After 30 days & $798 \pm 452 *$ & $168 \pm 100 *$ \\
\hline After 60 days & $775 \pm 382 *$ & $186 \pm 104 *$ \\
\hline
\end{tabular}

$* \mathrm{p}<0,05$ 
Figure 1. Ultrasonographic examination of foreharm skin before (A) and after (B) ESWT in one of the treated patients: similar findings were found in the other patients. A more regular skin structure is clearly present in panel B.

A

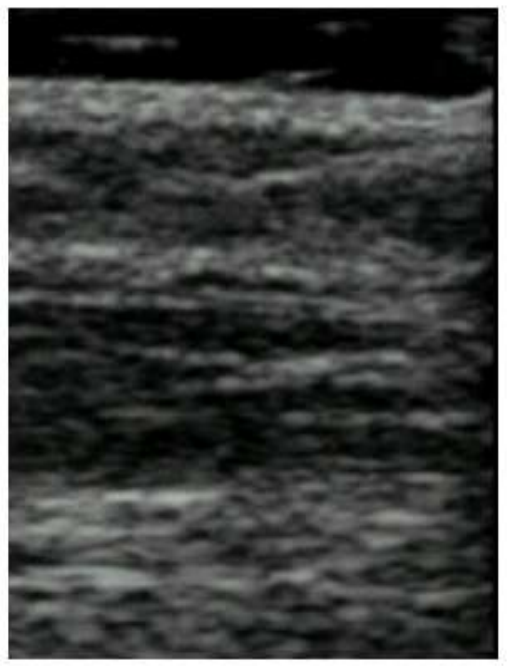

B

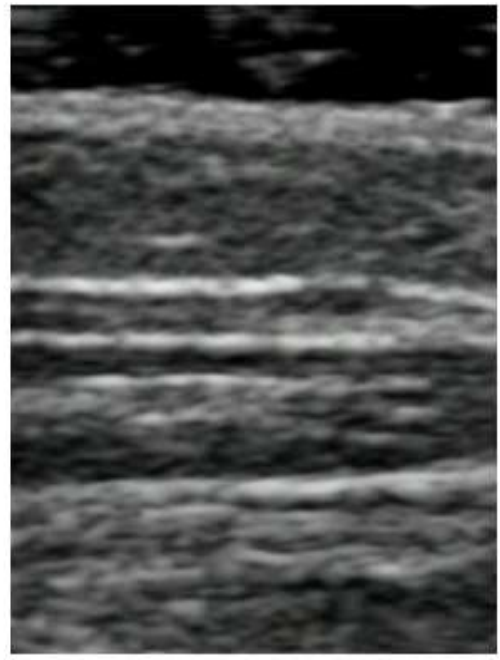

